\title{
Optimal Distributed Planning for Self Assembly of Modular Manipulators
}

\author{
Seung-kook Yun and Daniela Rus \\ Computer Science and Artificial Intelligence Laboratory \\ Massachusetts Institute of Technology, Cambridge, Massachusetts, USA \\ yunsk@mit.edu,rus@csail.mit.edu
}

\begin{abstract}
We describe algorithms to build self-assembling robot systems composed of active modular robots and passive bars. The distributed algorithms are based on locally optimal matching. We demonstrate how to build an active structure by the cooperative aggregation and disassembly of modular robotic manipulators. A target structure is modeled as a dynamic graph. We prove that the same optimality - quadratic competitive ratio - as for the static graph can be achieved for the algorithms. We demonstrate how this algorithm can be used to build truss-like structures.
\end{abstract}

\section{INTRODUCTION}

We wish to develop modular robots capable of construction tasks that integrate robotic elements and raw materials from the environment to create dynamic and controllable complex objects. In our previous work [1], [2] we describe a mechanism and supporting algorithm for the self-assembly of linkages that alternate 3 DOF robot modules called Shady3D with rigid bars. The resulting assemblies are controllable using distributed inverse kinematics protocols to achieve pick and place tasks. In this paper we extend the algorithm for self-assembling linkages in [2] to the self-assembly of arbitrary truss structures consisting of rigid bars and Shady3D-like robots with 3 rotational DOFs that are capable of grasping the bars on both ends. We assume a cache of robots and a cache of rigid bars. The robots know the goal shape but they do not know about each other. They are only capable of detecting each other and communicating locally, when they are in close proximity of each other. We show that this problem can be reduced to a distributed matching problem and analyze how sensing and communication can be used to guarantee that the robots construct the goal structure in an optimal number of steps. We then describe an implementation of this algorithm in simulation. Discussing its performance with a physical system will be considered in future.

The robot abstraction used by this algorithm is modeled on the Shady3D robot [1]. We assume that the robot looks like a rigid bar. The robot has grippers at both ends and is capable of grasping both rigid bars and robot units. The grippers can rotate. An additional rotational degree of freedom in the middle of the robot allows it to twist. This type of robot uses its grippers as feet to move in a truss-like environment. The truss provides the grounding support for each robot and for the truss-like assemblies the robots can create by grasping rigid bars. Thus, the scope of this work is restricted to truss construction in truss-like environments. Applications range from self-assembling scaffolds for construction to underwater bridges and space structures.

\section{A. Related work}

The robot arms we propose are closely related to truss climbing robots such as Staritz et al's "Skyworker" [3], Amano et al's handrail-gripping robot for firefighting [4], Ripin et al's pole climbing robot [5], Nechba, Xu, Brown et al's "mobile space manipulator SM2" [6], Kotay and Rus' "Inchworm" [7], and Almonacid et al's parallel mechanism for climbing on pipe-like structures [8] and our own Shady2D and Shady3D modules [1]. Also, we draw from an extensive body of literature on distributed motion planning [9], [10] and graph algorithms for matching [11] and flow [12].

\section{B. Outline}

This paper is organized as follows. Section II reviews the capabilities of Shady3D, the robot module we use in this work. Section III presents a goal of this work and formulates the problem. Distributed algorithms to build an active structure is described in Section IV. The optimality of the proposed algorithms is analyzed in Section V. The algorithms are implemented to build a robot hand-style structure in Section VI.

\section{EXPERIMENTAL INFRASTRUCTURE: SHADY3D AND RIGID BARS WITH LEDS}

In this work, we use Shady3D [13] as an active module and a bar with embedded IR LEDs as a passive one. The algorithms presented here depend on the abstract capabilities of Shady3D and can be instantiated on any other robot module with similar capabilities. We introduce the hardware and show how it self-assembles a tower

\section{A. Shady3D and passive bar}

Shady3D was originally designed with the goal of climbing 3-dimensional trusses as a first step toward tree-climbing robots. It has three joints for 3-D motion and two grippers on each side as shown in Figure 1(a). By connecting two Shady3Ds with a truss element as a medium of connection as in Figure 1(b), we can generate a 6DOF linkage. A passive 


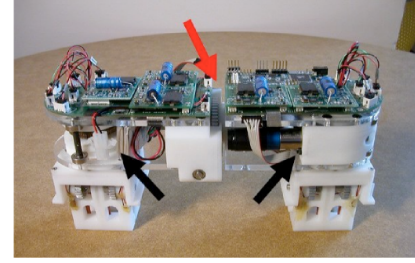

(a)

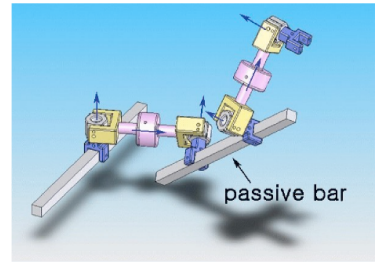

(b)
Fig. 1. (a) Shady3D and its structure: 3 -joints and 2 grippers. The arrows point to the locations of the joints. (b) A 6DOF one with inserting a passive bar between two robots [1]

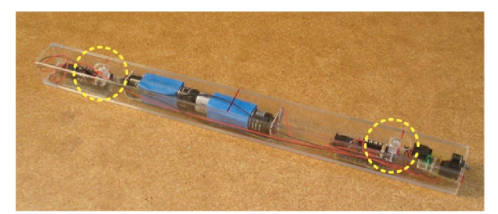

(a)

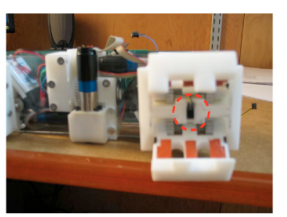

(b)
Fig. 2. (a) A passive bar with embedded IR LEDs (b) an IR sensor attached beneath the gripper [1]

bar emits IR signals via the IR LEDs embedded in the bar is shown in Figure 2(a).

\section{B. Self-assembled linkage: walking tower}

Multiple Shady robots can connect to one another using passive bars to form a larger active structure. Figure 3 shows snapshots of the self-assembly of a truss tower. Twelve active modules and eight passive bars are employed to build a threedimensional tower that can reconfigure itself by controlling active parts. Note that the robots are controlled by just a given sequence of motions - designed by hand. We will implement the same structure in Section VI by the proposed algorithm in a distributed way.
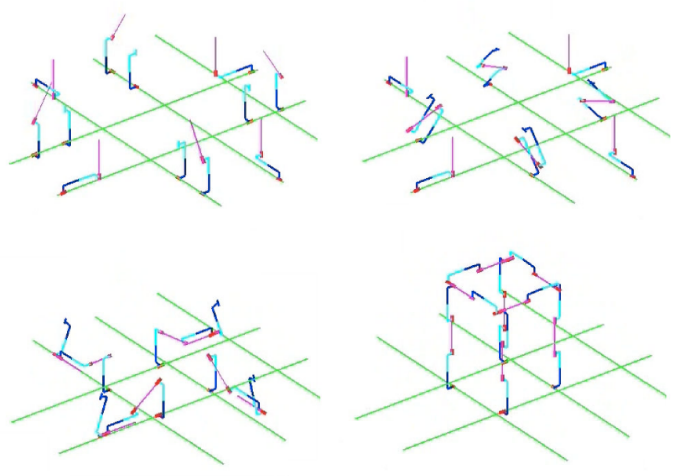

Fig. 3. Four snapshots of the tower building simulation. The Shady3D robot modules are drawn as an elongated U-shapes with light and dark halves; the free bars and the grid are drawn as straight segments [14].

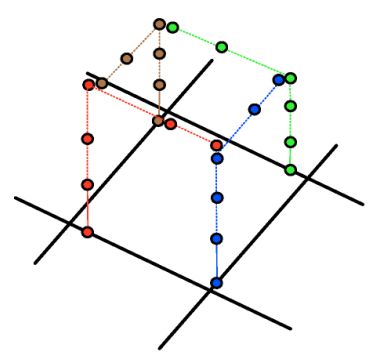

Fig. 4. A graph representation of the tower. Only the target nodes are shown. Circles are nodes, and non-activated and activated edges connect them. The graph has 4 on-truss and 16 non-truss target nodes. Each color denotes one of four trees.

\section{PROBLEM Formulation}

Our goal is to build an active structure composed of Shady3D-like robots and passive bars. We extend the algorithms we proposed in [15], where we consider how to optimally place a group of robots on a truss, to how the robots can create truss-like structures by self-assembly. Local information only will be used to coordinate robots to reach their designated good locations.

Let $A$ be a target to be assembled, $A$ can be represented as a graph as shown in Figure 4. The graph has active (solid lines in Figure 4) and non-active (dotted lines in Figure 4) edges. The nodes that are reachable to the robots are connected by active edges. The nodes have two types: a truss node is a part of the existing scaffold while a non-truss node is at the growing robotic truss. The graph begins as a connected system that marks the initial truss/scaffold for the assembly. As a robot learns that some of the non-truss nodes are occupied, its graph is updated by adding edges connected to the nodes. Every non-truss node should be linked from a on-truss node by non-activated edges. We call such a node (on a truss) a root, and the nodes that rooted at the node form a tree structure. For example, the tower in Figure 4 consists of four trees which is denoted by different colors.

To formulate the problem, we make the following assumptions and notations. We inherit many of them from [15].

- We are given a 3D structure with known geometry. The structure is modeled as a dynamic undirected graph $G$, whose vertices are points where a robot can grasp if the connecting edges are active. There is a positive cost on each edge.

- Passive bars are reachable from any root nodes.

- Each robot is modeled as two points that corresponds to an anchor and non-anchor gripper on the graph G.

- There are $k$ identical robots on the truss.

- All the robots start at on-truss nodes.

- Each robot can communicate with the robots that occupy adjacent nodes.

- The set of initial pairs of nodes in the graph is $R$; robot $i$ is initially located at $r_{i}$. These locations are not known to the robots.

- The set of target pairs of nodes is $T ; T=\left\{t_{1}, t_{2}, \ldots, t_{k}\right\}$ 
- The goal structure is feasible; given starting locations, there exist a sequence to build it.

The second assumption can be realized when passive bars are supplied at specific locations around roots by other robots or machine. For more general case, we will consider that bars are supplied at any position, as future work. Local commnuincation is a reasonable assumption since widerange communication may spend large amount of energy which is not allowed for a modular robot with a limited power source.

\section{Distributed Algorithm to Build an Active Structure By LOCALly Optimal Matching}

In this section we propose algorithms for the distributed placement of $k$ robots to build an active structure using distributed locally optimal matching. Our problem can be defined as finding a matching between $R$ and $T$, where $T$ can include non-truss nodes.

Each robot runs local algorithms for single robot's locomotion as well as self assembly and disassembly of multi robots. In particular, we focus on how to resolve collisions between robots, because they lead to co-operative reconfigurations and self-assembly.

Each robot's state includes the following data:

- ID: identification number

- Mode: Single if alone, Multi if in a tree

- Communicating: true it is communicating with others

- Status: what it is doing now. Idle, Busy, Move, Settled, or Assembling

- Location: currently occupying nodes by the anchor and the non-anchor gripper

- Position: only for MULTI mode, ROOT if it is a root of a tree and $L E A F$ otherwise

- Initial and target nodes pair

- Match list: a list of the initial and target nodes pairs it has learned by the collisions only with settled-down robots.

- Root-sided robot and Leaf-sided list: a list of which robots are connected to me and how they are connected. There is only one root-sided robot.

- Multi Job: a task for a root robot to do

When a robot is in Multi mode in a tree, a root robot of the tree has a role of a local brain to communicate with other robots in the tree and decide what the tree should do.

\section{A. Algorithm overview}

Figure 5 shows an expected sequence of building two trees (e.g. columns of a bridge). Each tree is composed of two robots and a passive bar. The following steps are required computationally to carry out this distributed assembly.

- Locate a robot on a truss by locomotion

- Add a unit to a tree

- Cut a unit from a tree

- Control trees to do above tasks

The following sections detail the phases of the algorithm.

\section{B. Deployment}

Algorithm1 shows the main control loop. After initialization, each robot executes the distributed deployment algorithm (Algorithm 2 or 3) according to its mode, unless the robot is communicating. Otherwise, it handles messages from the communicating robot. Afterward, it updates its mode and position based on its state.

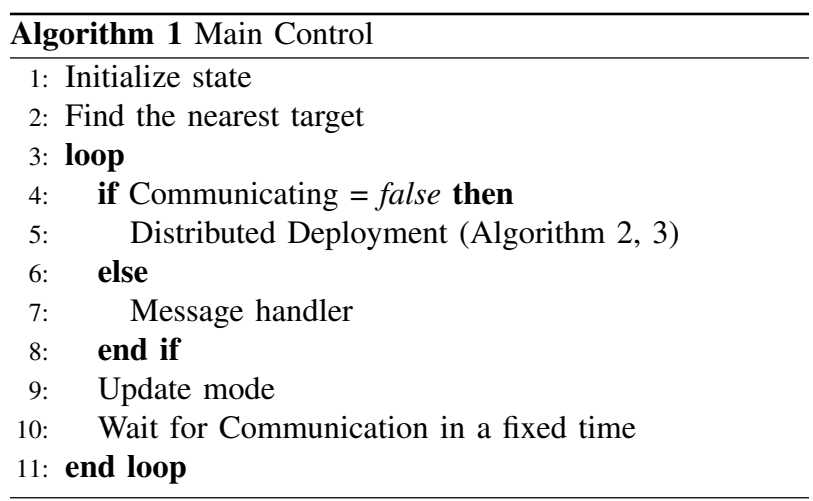

Algorithms 2 and 3 are the procedures that enable a given robot with a target construction tree to move, detect collisions and handle them. The first algorithm checks if this robot has arrived at its desired target. If so, it sets Status=Settled, and stops. Otherwise, the robot checks for collisions by communication. If the next node is empty, it takes a step and updates the resources. In case of a collision the robot calls the collision handler (Algorithm 4).

For multi-mode robots, only the root robot checks a given job and executes it. Currently, we have only one case for cutting a leaf of the tree, but other behaviors can be added (e.g. locomotion of a tree).

\section{Handling Collisions}

The collision handler (Algorithm 4) starts with trying communication to a blocking robot, ID of which is noted as $c I D$. $\operatorname{SEND}(I D$, message $)$ is a command to transmit the message to a robot that has the ID. This command works only with neighborhood robots. If the collided robot allows communication by sending the message, the handler calls the detailed collision handler. After the detailed handler ends, the algorithm finishes communication by sending the message ENDCOMM, if the robot is still communicating.

Algorithm 5 is the detailed handler. We have found out that the algorithm for Single mode (developed in [15],) can be applied for a tree by considering a leaf of the tree as a Single mode robot. There are some special cases only for Multi mode such as adding and cutting a leaf, which will be explained in Section IV-D.

1) Crossing path: When the paths of a robot and the blocking robot cross each other, the two robots exchange their destinations by exchanging some of their state. When the colliding one is a tree, the same rules apply for its leaf to cut. 


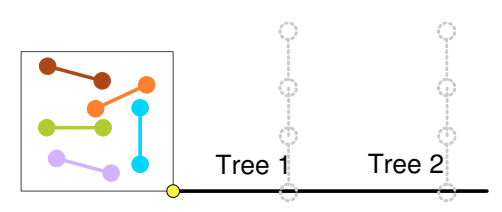

(a)

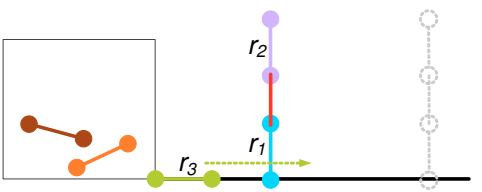

(d)

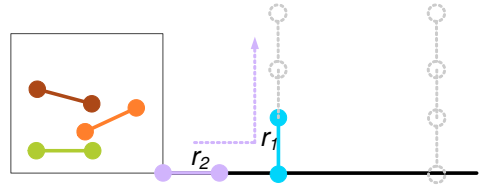

(b)

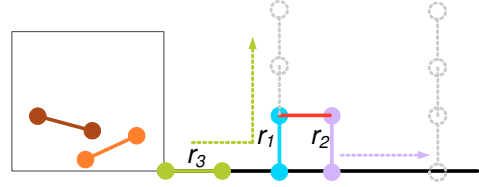

(e)

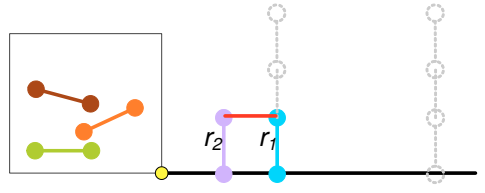

(c)

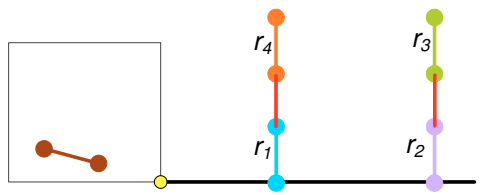

(f)

Fig. 5. Building two trees by four Shady3D-like robots. (a)Robots are in a reservoir with a given design of the columns. The goal structure is denoted by gray dotted lines. In the beginning, every robots goes to the root of the first tree since it is the nearest target. (b) $r_{1}$ occupies the root of the first tree. $r_{2}$ collides $r_{1}$, and finds the next optimal target. (c) $r_{2}$ is being added to $r_{1}$. (d) $r_{3}$ also collides $r_{1}$, and finds the next target (the root of the second tree). (e) $r_{2}$ goes to the second root instead of $r_{3}$, and is being cut from $r_{1}$. (f)Sequentially, all the target nodes are occupied by four robots.
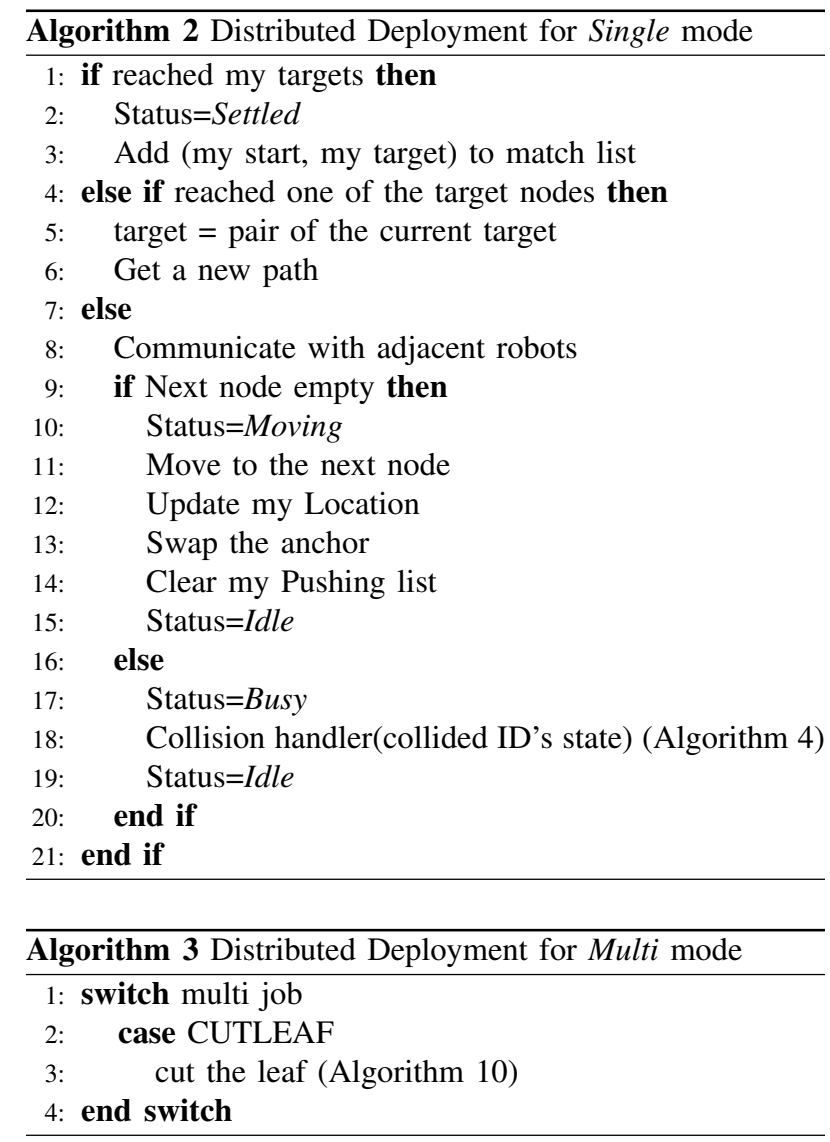

2) Breaking a Deadlock: A deadlock is a status in which some robots can not move even though their paths do not cross. In this case, the robot with lowest ID forces the blocking robot to execute Exchanging the robots until a robot in the list does not block it anymore. The details are in [15].

3) Finding a new target: How to select a new target when a target node of a colliding robot is in the collided robot's tree is important for optimal matching. To solve this conflict,
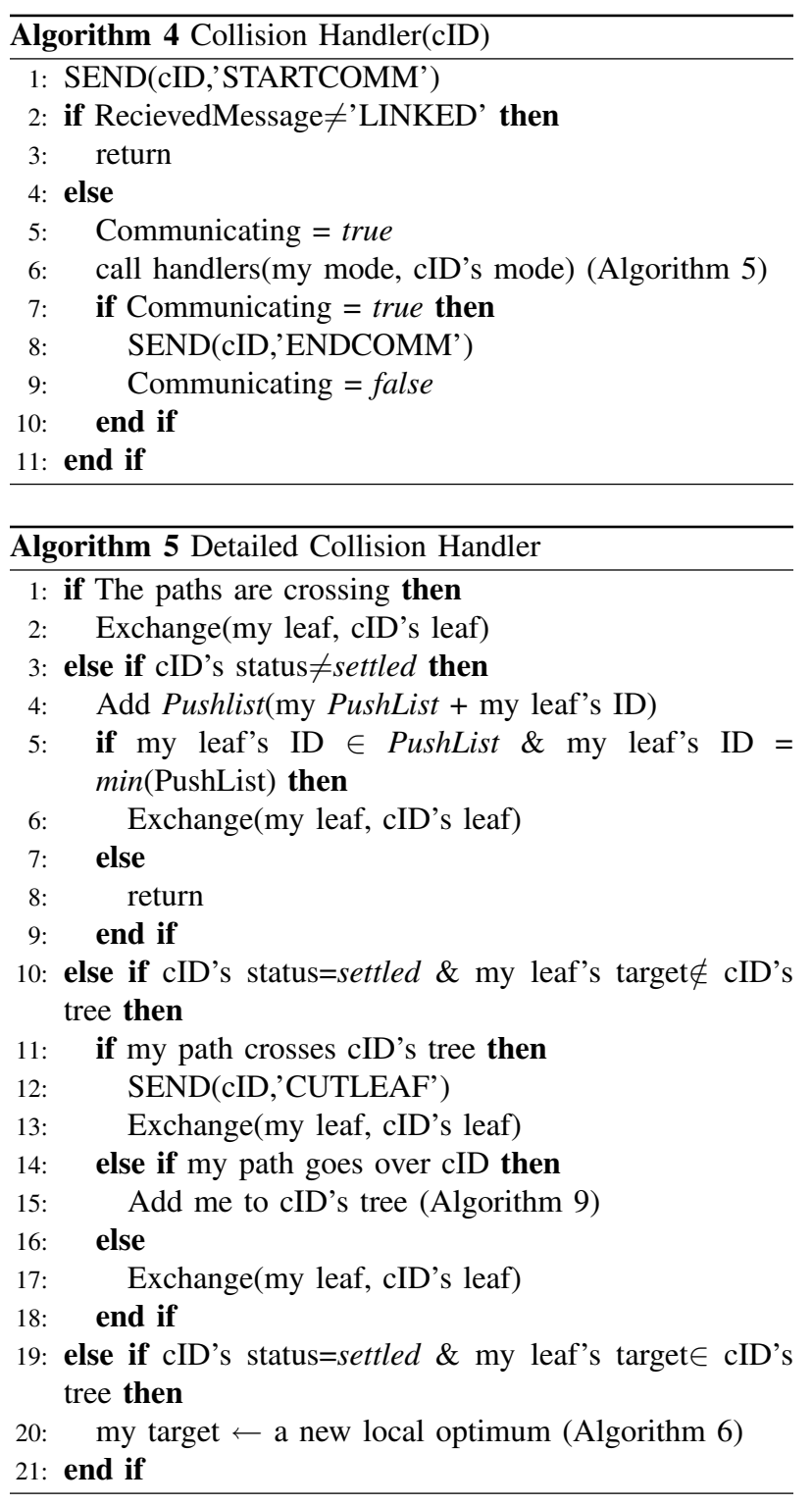
the blocked robot finds another target node, computes a new path to it, and follows the path as it has done. The new target is a node in the optimal set of the target nodes matched with the initial nodes of the blocked robot and the match lists of both robots (or trees).

Algorithm 6 describes the locally optimal matching. When robot $p$ has found that robot $q$ occupied its target, they merge their match list $\left\{R_{q}, T_{q}\right\}$ and $\left\{R_{p}, T_{p}\right\}$. Using the merged match list and its starting node, it calculates the next target which is incident on the locally optimal matching $M_{p+q}$.

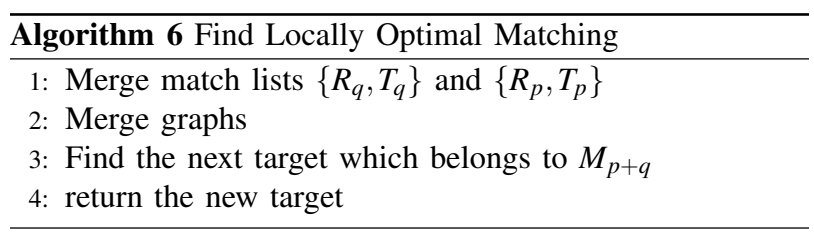

D. Multi-robot movement for self-assembly: adding and cutting a leaf

When a robot collides with a settled tree, a leaf of the tree should move instead of the root, and the tree should execute adding and cutting the leaf. We have seen a sequence of handling collision between a robot and a tree in Figure 5. When the target of a robot is on the next empty nodes of the tree(Figure 5(b), ) line 14 of Algorithm 5 is called to add the robot to the tree. The robot is added to the tree, connected by the red passive bar (Figure 5(c)). When the path of a robot is crossing the tree (Figure 5(d)). The robot requests to cut a leaf from the tree according to line 11 of Algorithm 5, and it exchanges its identity with the leaf. At the next turn of the main control loop, it has the target of the leaf, while the leaf is being cut (Figure 5(e)).

To implement these behaviors, communication along a tree is required. We call it SENDTREE as in Algorithm 7, where a simple depth-first search algorithm is used with the state of leaf-sided robot list and root-sided robot. A robot in the tree propagates a received message from its root-sided robot to leaf-sided ones, waits for the answer of the leaf-sided, and finish communication with the root-sided.

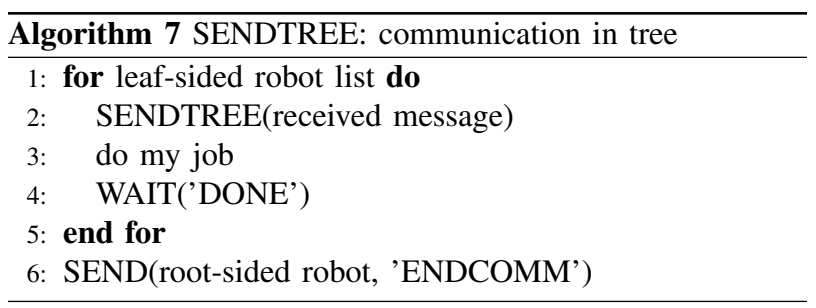

1) Add a leaf: When a tree adds a leaf, the robot uses Algorithm 8, while the new leaf works with Algorithm 9 in parallel. When the new leaf sends the message 'ADDLEAF', the root checks if a bar is connected to the new leaf's target node. If the bar does not exist, the tree makes a path to a new bar by inverse kinematics. The path consists of new locations of the nodes in the tree, from the root to a side of the bar. The root robot sends the path to the others in the tree, and robots that are on the path move with the given locations. The root commands to grasp the bar, and calculates another path to the anchor node of the new leaf. Afterward, the root sends the corresponding locations to the new leaf, while the tree moves with the new path. They exchange some messages to synchronize grasping and releasing, and end communication. Note that communication finishes before the addition is completed. Otherwise the leaf may move far away from the communication range. Finally, the tree returns to its original locations and updates the match list and the graph.
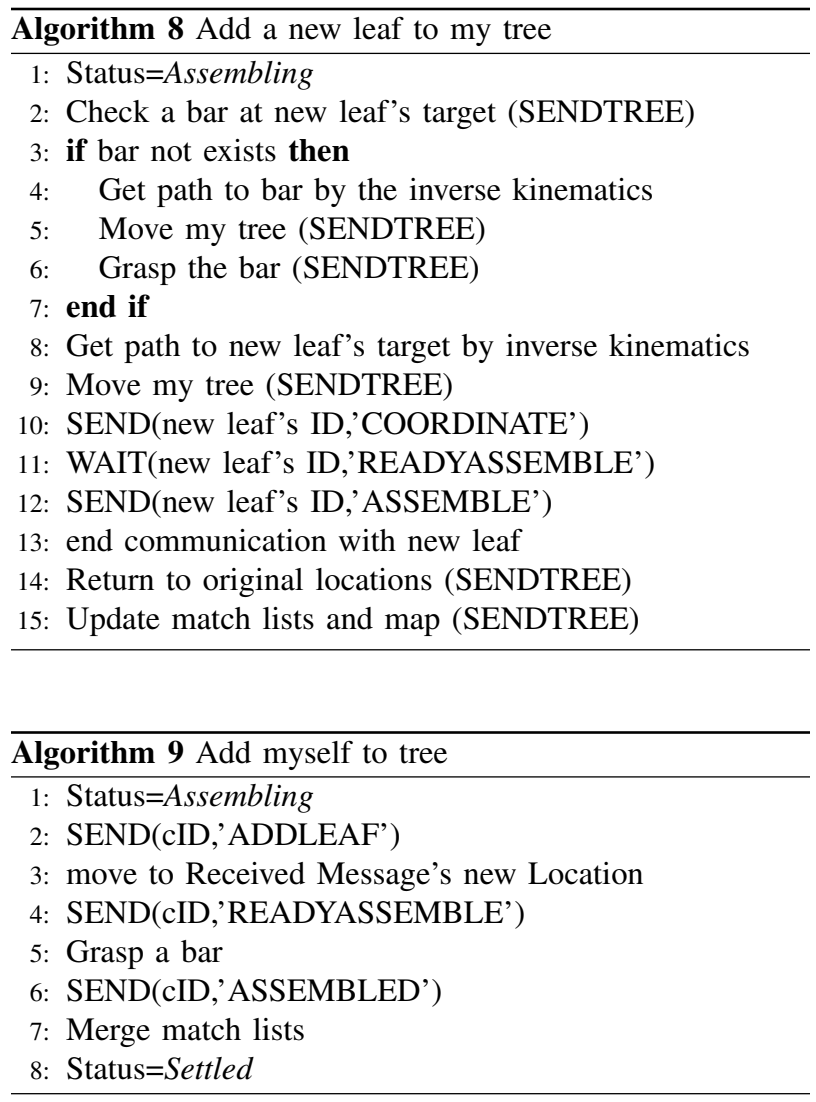

2) Cut a leaf: Adding a leaf happens between colliding robots. Cutting a leaf is a procedure between robots in a tree, and is designed as an independent process. Cutting a leaf is called when a root robot has state MultiJob=CUTLEAF (Algorithm 3), which is triggered by the colliding robot (Algorithm 5). Algorithm 10 shows how cutting a leaf is implemented at the root. The root begins to cut a leaf as it finds a next node on a truss where the leaf should be located. If the node is empty, the root gets a path to it by inverse kinematics, reconfigures the tree, grasps the node, and release the leaf from the tree. Otherwise the collision handler is called.

\section{ANALYSIS}

In this section, we briefly review the previous analysis of the optimality for distributed matching [15]; the distributed 


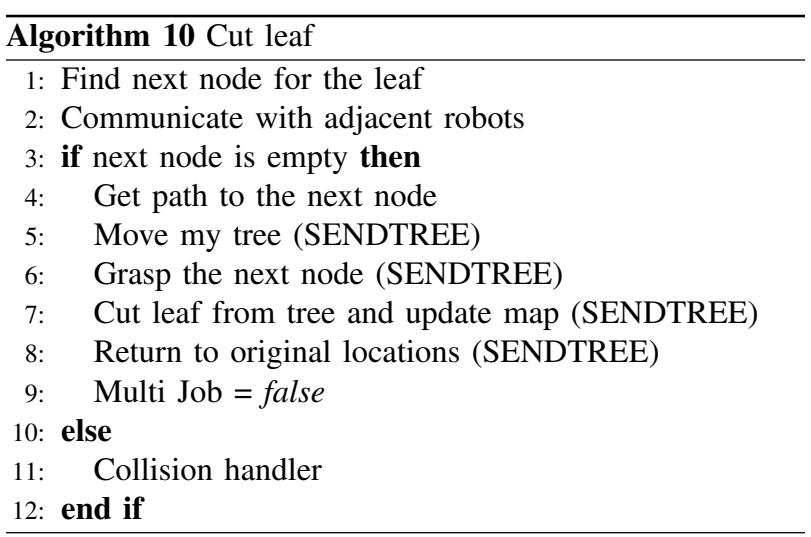

algorithm was shown to have $O\left(k^{2}\right)$ asymptotic competitive ratio to the global optimum, wheares a greeday algorithm which seeks the nearest next target has an exponential competitive ratio. We prove that the same bound still holds for our dynamic graph.

\section{A. Optimality of the distributed matching for a static graph}

In [15], our analysis used several results on minimal weight partial matching from [11]. The minimal weight partial matching $M_{i}$ which is the set of edges that form the minimal weight partial perfect matching between the subset $\left\{r_{1}, r_{2}, \ldots, r_{i}\right\}$ and subset of $T$ with a minimal number of edges in $M_{i}-M_{i-1}$. Let $T_{i}$ be the subset of $T$ consisting of vertices of $T$ which are incident on $M$.

Lemma 1: The cost of the $M_{i}$ s form a monotonically nondecreasing sequence.

Lemma 2: For each $i$, the set difference $T_{i}-T_{i-1}$ contains exactly one vertex.

Proofs of Lemma 1- 2 are given in [11]. We extended the partial optimal matching results to our algorithm.

Lemma 3: The initial node and target node matched by a settled down robot stays fixed.

Lemma 4: A merged matching list from the lists of two robots has the exactly same number of initial nodes and target nodes.

Lemma 5: The distributed deployment algorithm has $O\left(k^{2}\right)$ asymptotical competitive ratio.

Proofs of Lemma 3- 5 are given in [15].

\section{B. Optimality for the dynamic graph}

The key idea for proving the competitive ratio of the distributed matching for dynamic graphs is that a non-truss node should have its root on the truss. This implies that there must exist a closer root node from a robot than any non-truss nodes.

Lemma 6: The locally optimal matching only includes connected target nodes in a given dynamic graph. i.e. it never has target nodes that do not have any activated edges.

Proof: We use induction. When a robot starts, the proof is trivial. Suppose the lemma holds for $k-1$ robots and it finds a new target $t_{k}$, not connected in its graph, with the locally optimal matching $M_{k}$. Let us say $r_{k}$ is matched to
TABLE I

COUNT OF THE OPERATIONS

\begin{tabular}{|c||c|c|}
\hline Count of operations / Structure & Hand & Tower \\
\hline Total move/Optimum & $297 / 262$ & $84 / 66$ \\
Average communication & 40 & 8.3 \\
Exchange & 70 & 6 \\
Getting a new optimum & 36 & 18 \\
Adding a leaf & 34 & 10 \\
Cutting a leaf & 22 & 2 \\
\hline
\end{tabular}

$t_{k}$ in $M_{k}$. Note that we can always find a connected target node $\hat{t}_{k}$, in a tree that includes $t_{k}$, which are closer to a truss than $t_{k}$, because $t_{k}$ should be connected to a truss by a tree with $\hat{t}_{k}$. Now we have the better matching $\hat{M}_{k}$ by coupling $r_{k}$ to $\hat{t}_{k}$ with maintaining the other matching in $M_{k}$. It is contradiction. Therefore the lemma holds for $k$ robots.

Lemma 7: The distributed matching algorithm for our dynamic graph has the same competitive ratio $O\left(k^{2}\right)$ as that for a static graph.

Proof: By Lemma 6, the locally optimal matching will find a new target that should be connected in the given graph, no matter the algorithm uses a fully connected graph or the given one. Therefore, the algorithm has the same competitive ratio as that uses a static graph as in [15].

\section{IMPLEMENTATION}

We have implemented the distributed placement algorithm to build an active structure in Java. We simulated each robot as an independent process (thread) to ensure parallelism.

Figure 6 shows snapshots of building a hand on $\mathrm{H}$ structured trusses. 18 robots are deployed and passive bars are around the root nodes. Yellow and green circles denote each gripper of a robot, and pink bars are passive bar. Small red circles are target nodes which compose five trees. Robots start from side trusses and gather into the center as they perform successive add-leaf and cut-leaf operations. For a better view, passive bars appear only when they are grasped. 13 bars are used to connect the robots.

Figure 7 is implementation of building a tower we suggested in II-B. The tower consists of four trees, each of which has 3 robots and 2 bars. Note that the implementation of the tower in II-B was done by a central controller that knows the exact sequence of motions generated by hands.

The brief statistical summary of the simulations is in Table I. Collision among trees is ignored, and will be considered in our future work.

\section{CONCLUSION}

This paper describes a unified approach to implement building an active structure by self-assembly using a dynamic graph and distributed matching. We developed this work in the context of a modular mobile manipulator Shady3D. The algorithm works in a distributed way so that robots depend on only local information. A target structure is modeled as a dynamic graph with edges that are not activated until one of the incident nodes is occupied by a robot. The robots discover their locations in the structure incrementally 


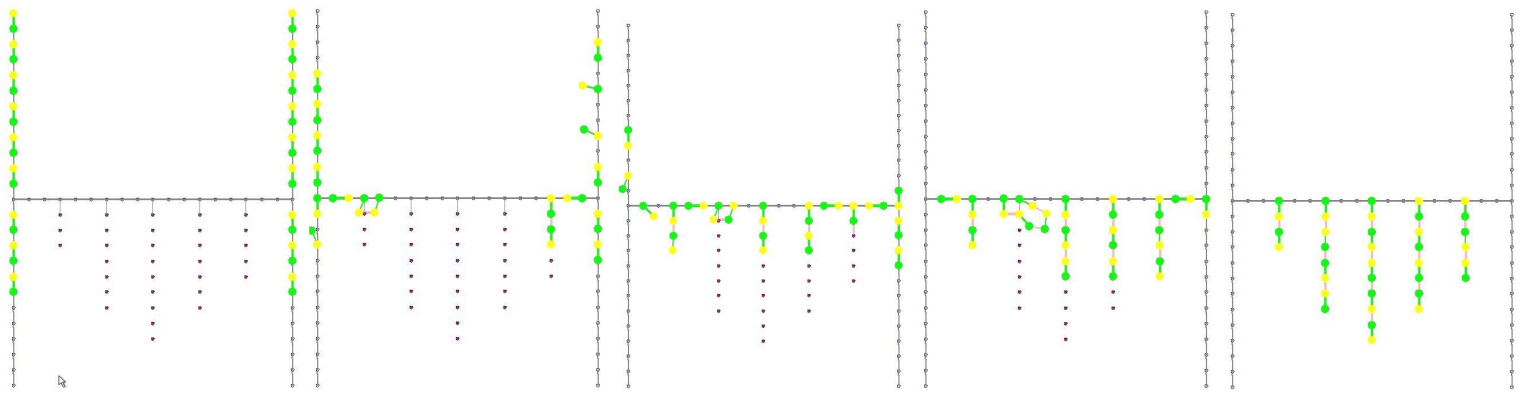

Fig. 6. Snapshot of building a hand-like active structure. Thick gray lines are trusses, while thin ones are edges to connect non-truss nodes. 18 robots and 13 passive bars are connected. Yellow circles are the left grippers and greens are the right ones. The bars are pink.
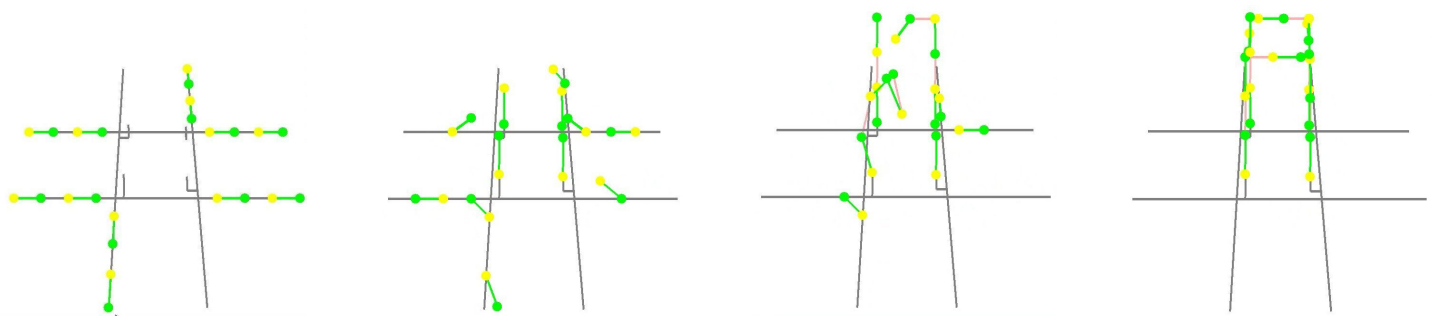

Fig. 7. Snapshot of building a tower we proposed in II-B. 12 robots and 8 passive bars are used.

through collisions, and update their graph. Self-assembly of the robots makes a tree with robots and bars, and a root of the tree becomes a local brain to control the leaves with the node-based inverse kinematics. Adding and cutting a leaf are implemented by communication between a robot to be added or cut and the root of the tree. The robots build the structure in a locally optimal way, and we have proved that the same competitive ratio for a static graph holds for our dynamic graph. We implement the proposed algorithms in building a robot hand with five fingers by 18 robots and 13 bars.

Much work to be done remains such as implementation on hardwares, collision avoidance and robust communication protocols.

\section{ACKNOWLEDGEMENTS}

This project has been supported in part by Boeing Corporation and NSF awards 0735953 and 0707601. Seungkook Yun is supported in part by Samsung Scholarship. We are grateful for the supports. We thank Marsette Vona and Carrick Detweiler for their help and insightful discussions.

\section{REFERENCES}

[1] Carrick Detweiler, Marsette Vona, Yeoreum Yoon, Seung-kook Yun, and Daniela Rus, "Self-assembling mobile linkages," IEEE Robotics and Automation Magazine, vol. 14(4), pp. 45-55, 2007.

[2] Seung-kook Yun and Daniela Rus, "Self assembly of modular manipulators with active and passive modules," in Proc. of IEEE/RSJ IEEE International Conference on Robotics and Automation (to appear), Pasadena, CA, May 2008.

[3] Peter J. Staritz, Sarjoun Skaff, Chris Urmson, and William Whittaker, "Skyworker: A robot for assembly, inspection and maintenance of large scale orbital facilities," in IEEE ICRA, Seoul, Korea, 2001, pp. $4180-4185$.
[4] Hisanori Amano, Koichi Osuka, and Tzyh-Jong Tarn, "Development of vertically moving robot with gripping handrails for fire fighting," in Proceedings of the 2001 IEEE/RSJ International Conference on Intelligent Robots and Systems, Maui, HI, 2001, pp. 661-667.

[5] Zaidi Mohd Ripin, Tan Beng Soon, A.B. Abdullah, and Zahurin Samad, "Development of a low-cost modular pole climbing robot," in TENCON, vol. I, Kula Lumpur, Malaysia, 2000, pp. 196-200.

[6] Michael Nechyba and Yangsheng Xu, "Human-robot cooperation in space: SM2 for new space station structure," IEEE Robotics and Automation Magazine, vol. 2, no. 4, pp. 4-11, Dec. 1995.

[7] Keith D. Kotay and Daniela L. Rus, "Navigating 3d steel web structures with an inchworm robot," in Proceedings of the IEEE International Conference on Intelligent Robots and Systems, 1996.

[8] M. Almonacid, R. J. Saltarén, R. Aracil, and O. Reinoso, "Motion planning of a climbing parallel robot," IEEE Transactions on Robotics and Automation, vol. 19, no. 3, pp. 485-489, 2003.

[9] Z. Butler and D. Rus, "Distributed planning and control for modular robots with unit-compressible modules," International Journal of Robotics Research, vol. 22, pp. 699-715, 2003.

[10] W. Lee and A. Sanderson, "Dynamic analysis and distributed control of the tetrabot modular reconfigurable robot system," Autonomous Robots, vol. 10(1), pp. 67-82, 2001.

[11] B. Kalayanasundaram and K. Pruhs, "Online weighted matching," Journal of Algorithms, vol. 14(3), 1993.

[12] J. Edmonds and R. Karp, "Theoretical improvements in algorithmic efficiency for network flow problems," Journal of ACM, vol. 19, pp. 248-264, 1972.

[13] Y. Yun and D. Rus, "Shady3d:a robot that climbs 3d trusses," in Proceedings of the IEEE International Conference on Robotics and Automation, pp. 4071-4076.

[14] Y. Yoon, "Modular robots for making and climbing 3-d trusses," Master's thesis, Computer Science and Artificial Intelligence Laboratory, MIT, Cambridge, 2006.

[15] Seung-kook Yun and Daniela Rus, "Optimal distributed planning of multi-robot placement on a 3d truss," in Proc. of IEEE/RSJ International Conference on Intelligent Robots and Systems, Oct. 2007. 